\title{
Correlation of total reference air-kerma (TRAK) to prescription isodose surface volume in vaginal cylinder high-dose-rate brachytherapy
}

\author{
Ravindra Yaparpalvi, MS ${ }^{1,2}$, Keyur J. Mehta, MD ${ }^{1.2}$ \\ 'Department of Radiation Oncology Montefiore Medical Center, Bronx, New York, USA, ²Department of Radiation Oncology Albert Einstein \\ College of Medicine, Bronx, New York, USA
}

\begin{abstract}
Purpose: The purpose of this technical note was to investigate correlation of total reference air-kerma (TRAK) with volume enclosed by the prescription isodose surface in vaginal cylinder high-dose-rate (HDR) brachytherapy.

Material and methods: Treatment plans of 175 gynecological cancer patients treated at our institution with iridium-192 ( ${ }^{192}$ Ir) HDR brachytherapy using a single-channel vaginal cylinder applicator were retrospectively reviewed. Applicator size in diameter ranged from $20 \mathrm{~mm}$ to $40 \mathrm{~mm}$. Treatment length ranged from $30 \mathrm{~mm}$ to $90 \mathrm{~mm}$ (median, $50 \mathrm{~mm}$ ). Brachytherapy fractional dose was $5 \mathrm{~Gy}$ (Dose Ref $_{\text {f }}$ ) prescribed to $5 \mathrm{~mm}$ distance from cylinder surface. Parameters TRAK (cGy), source activity during treatment (Ci), total treatment time (s), and prescription isodose surface volume $\mathrm{ISV}_{\mathrm{Ref}}\left(\mathrm{cm}^{3}\right)$ were recorded from individual treatment plans. In each case, vaginal tissue volume $\left(\mathrm{V}_{\mathrm{VT}}\right)$ enclosed by prescription isodose was calculated by subtracting cylinder volume enclosed by the prescription isodose from ISV $\mathrm{Vef}_{\text {. }}$

Results: Total reference air-kerma correlated with the total volume enclosed by the prescription isodose via ISV $\mathrm{Ref}=$ $4768 \times\left(\right.$ TRAK$_{\text {Dose }} \mathrm{Ref}^{1.47}$. TRAK related linearly to the volume of vaginal tissue enclosed by the prescription dose via $\mathrm{V}_{\mathrm{VT}}=((138.3 \times \mathrm{TRAK})-8.2)$. Secondarily, TRAK related to the treatment time through time $(\mathrm{s})=882(\mathrm{~s} / \mathrm{cGy}) \times \mathrm{TRAK}$ (cGy), where 882 is (1/air-kerma strength) for $10 \mathrm{Ci}$ apparent activity of ${ }^{192} \mathrm{Ir}$ source.

Conclusions: The correlation of TRAK to the vaginal tissue volume encompassed by the prescription dose surface yields a useful predictive equation. The TRAK treatment time relationship enables quick verification of planned treatment time by knowing TRAK in any HDR brachytherapy application.

J Contemp Brachytherapy 2021; 13, 4: 447-450 DOI: https://doi.org/10.5114/jcb.2021.108599
\end{abstract}

Key words: TRAK, vaginal cylinder, vaginal tissue volume, HDR brachytherapy, treatment time, isodose volume, QA.

\section{Purpose}

Reporting of total reference air-kerma (TRAK) as a brachytherapy treatment parameter is recommended by the ICRU Report 89 [1]. TRAK is defined as the integral of reference air-kerma rate over the treatment duration, and summed for all source dwells (expressed in units, such as cGy at 1-meter reference distance). Historically, TRAK is related to the milligram-hour radium concept (radium mass $\times$ treatment time), and therefore represents intensity of treatment. Also, TRAK does not take into consideration geometry of brachytherapy application, and is considered an unambiguous physical quantity. While TRAK does not portray the shape of target volume treated nor predict dose distribution for a given application; however, dose to any point or volume is directly proportional to TRAK value [1]. In intracavitary brachytherapy, TRAK has been shown to correlate with volume enclosed by isodose surface (ISV). Accordingly, predictive mathematical expressions relating volume enclosed by isodose to TRAK and dose are suggested in literature [2-7].

In this study, we aimed to relate TRAK to volume enclosed by the prescription isodose surface $\left(\mathrm{ISV}_{\mathrm{Ref}}\right)$ in a single-channel vaginal cylinder (VC) high-dose-rate (HDR) brachytherapy. Since VC brachytherapy represents one of the most widely utilized gynecological brachytherapy applications [8], we felt it would be interesting to obtain TRAK-irradiated volume relationship and compare it to the published TRAK-volume relationship for intracavitary brachytherapy.

\section{Material and methods}

Brachytherapy treatment plans of 175 patients treated for gynecological cancer at our institution with iridium-192 ( ${ }^{192}$ Ir) HDR brachytherapy, using single-channel 
VC applicator (Elekta AB, Stockholm, Sweden), were retrospectively reviewed. VC size distribution was $20 \mathrm{~mm}$ $(n=4), 25 \mathrm{~mm}(n=12), 30 \mathrm{~mm}(n=52), 35 \mathrm{~mm}(n=104)$, and $40 \mathrm{~mm}(n=3)$. Treatment length ranged from $30 \mathrm{~mm}$ to $90 \mathrm{~mm}$ (median, $50 \mathrm{~mm}$ ). Brachytherapy dose (Dose Ref $_{\text {f }}$ ) of 5 Gy per fraction was prescribed at $5 \mathrm{~mm}$ depth from cylinder's surface. A commercial treatment planning system based on the AAPM TG-43 dose calculation formalism was utilized for dose planning and optimization (Oncentra; Elekta AB, Stockholm, Sweden). Source dwell times in each case were optimized using dose-point optimization, with dose points described laterally to the cylinder surface and superiorly at the cylinder dome. Source position separation (step-size) applied in treatment plans ranged from $2 \mathrm{~mm}$ to $5 \mathrm{~mm}$. Previously, with our HDR microSelectron v2 afterloader (Nucletron), the source step-size in increments of $2.5 \mathrm{~mm}$ was possible, whereas in our current Flexitron HDR (Elekta) afterloader, source position separations within $1 \mathrm{~mm}$ increments are possible. Either a $2.5 \mathrm{~mm}$ or $5 \mathrm{~mm}$ source step-size was applied in planning with microSelectron-HDR v2 afterloader, and a $2 \mathrm{~mm}$ or $3 \mathrm{~mm}$ source step-size was utilized with Flexitron HDR afterloader, which was achieved by activating every $2^{\text {nd }}$ or $3^{\text {rd }}$ dwell position. In general, finer step-sizes ( $2 \mathrm{~mm}$ or $3 \mathrm{~mm}$ ) were used for shorter treatment lengths $(\leq 50 \mathrm{~mm})$, and coarser step-size of $5 \mathrm{~mm}$ was applied in longer cylinder treatment lengths $(\geq 50 \mathrm{~mm})$. Patients were treated either with a microSelectron-HDR v2 or a Flexitron HDR treatment unit.

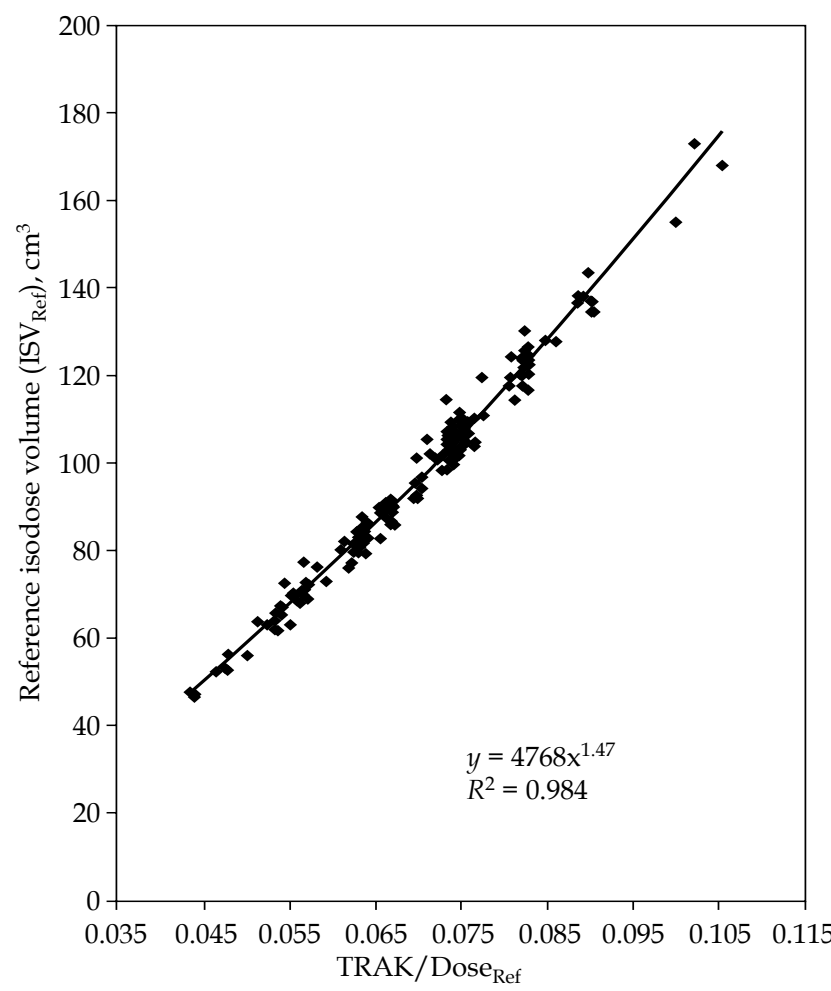

Fig. 1. A scatterplot of (TRAK/D $D_{\text {Ref }}$ ) vs. $I_{\text {Ref }}$ for 175 vaginal cylinder brachytherapy cases. The trend line is a power-law representation. TRAK - total reference air-kerma (cGy), Dose $e_{\text {Ref }}$ - prescription dose (Gy), $\mathrm{ISV}_{\text {Ref }}$ - volume covered by prescription isodose $\left(\mathrm{cm}^{3}\right)$
From each patient's brachytherapy treatment plan, the following parameters were extracted: TRAK (cGy at 1 meter) representing the fractional total reference air-kerma of brachytherapy treatment, planned source strength (in $\mathrm{Ci}$ ), total treatment time (in seconds), and isodose surface volume corresponding to prescription isodose $\left(\mathrm{ISV}_{\mathrm{Ref}}\right.$ in $\left.\mathrm{cm}^{3}\right)$ from a dose-volume histogram. Additionally, the volume of vaginal cylinder included in ISV $_{\text {Ref }}$ was calculated by contouring the vaginal cylinder length enclosed by the prescription isodose and obtaining the corresponding volume. In each case, real irradiated vaginal tissue volume $\left(\mathrm{V}_{\mathrm{VT}}\right)$ with prescription isodose was then calculated by subtracting the cylinder volume enclosed by the prescription isodose from $I_{\text {Ref }}$. For the purpose of the study, in each case, the planned total treatment was normalized to the time required for a $10 \mathrm{Ci}$ apparent activity ${ }^{192} \mathrm{Ir}$ source. Data were analyzed using basic statistics and graphing tools (Microsoft Excel).

\section{Results}

\section{TRAK vs. reference isodose surface volume}

A scatter plot of (TRAK/ Dose ${ }_{\text {Ref }}$ ) vs. ISV $_{\text {Ref }}$ is presented in Figure 1. TRAK exhibited a relationship with ISV $_{\text {Ref }}$ which was best described by a power-law type equation $\left(R^{2}=0.984\right)$ :

$$
\mathrm{ISV}_{\text {Ref }}=4768 \times(\text { TRAK/ Dose } \text { Ref })^{1.47} \quad \text { Eq. (1). }
$$

\section{TRAK vs. vaginal tissue irradiated volume}

A scatter plot of TRAK vs. $\mathrm{V}_{\mathrm{VT}}$ is presented in Figure 2. TRAK exhibited a relationship with $\mathrm{V}_{\mathrm{VT}}$, which was best described by a linear equation $\left(R^{2}=0.801\right)$ :

$$
\mathrm{V}_{\mathrm{VT}}=((138.3 \times \mathrm{TRAK})-8.2)
$$

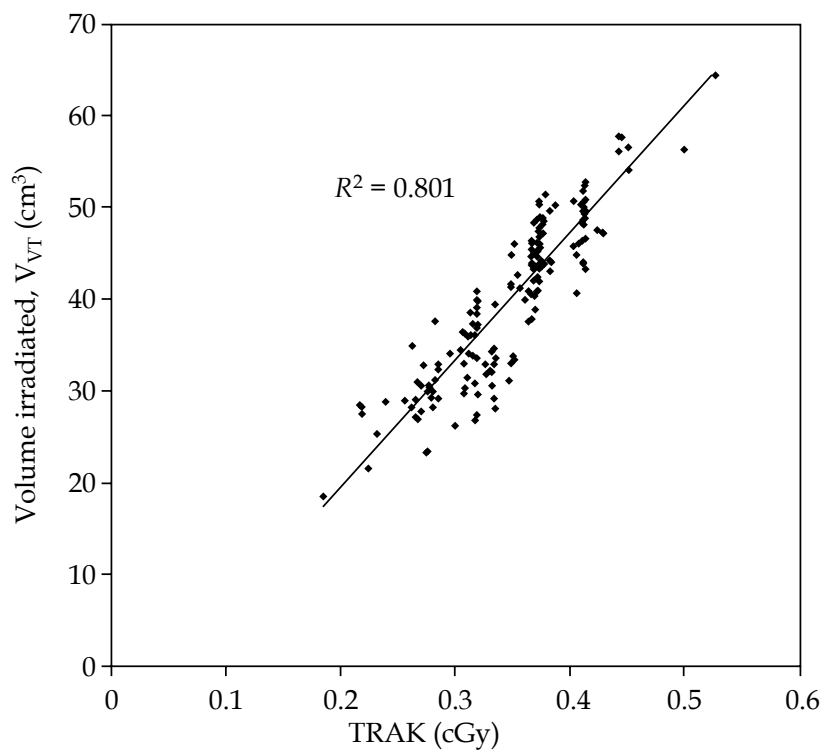

Fig. 2. A scatterplot of TRAK vs. $V_{V T}$ for 175 vaginal cylinder brachytherapy cases. TRAK - total reference air-kerma (cGy), $\mathrm{V}_{\mathrm{VT}}$ - volume of vaginal tissue covered by prescription isodose $\left(\mathrm{cm}^{3}\right)$ 


\section{TRAK vs. treatment time}

A scatter plot of TRAK vs. total treatment time is presented in Figure 3. TRAK related to treatment time was shown via a relation:

Time $(\mathrm{s})=882(\mathrm{~s} / \mathrm{cGy}) \times$ TRAK $(\mathrm{cGy}) \quad$ Eq. (3),

where 882 is (1/air-kerma strength) for a 10-Ci apparent activity ${ }^{192}$ Ir source.

\section{Discussion}

The power-law relationship between TRAK and the volume enclosed by the prescription isodose surface based on our study data (Eq. 1) yielded a predictive equation, which is similar to the one proposed by Wilkinson and Ramachandran [2]. In their study, the authors proposed that the spherical volume covered by any dose level can be calculated by the expression: $\mathrm{V}\left(\mathrm{cm}^{3}\right)=4965$ (TRAK/ $\left.D_{\text {Ref }}\right)^{3 / 2}$, presuming that the total activity is concentrated into a point-source and distance following inverse square law for dose gradient. While the ICRU 89 Report [1] recommends reporting of TRAK as a parameter, ISVs in cervix brachytherapy could be utilized for reporting treatment intensity and for comparing treatments across fractionation schedules [6, 9]. In VC brachytherapy, the irradiated surface volume at the prescription dose level also includes the volume of VC utilized for the patient, and does not strictly corresponds to the irradiated tissue volume. It is not a standard universal practice in clinical VC brachytherapy to routinely adapt contouring of target volume during treatment planning. This is mainly due to differences between practitioners on where the dose is prescribed (at surface vs. at depth), and also because of differences in dose optimization methods utilized $[8,10]$. In our opinion, this limits the clinical utility of reporting $\mathrm{ISV}_{\text {Ref }}$ in VC brachytherapy. Interestingly, in our study, we observed that TRAK data correlates linearly with the volume of vaginal tissue covered by the prescription dose (Figure 2; Eq. 2). Various studies on VC brachytherapy have shown correlations between total dose, dose per fraction, vaginal length treated, and vaginal late toxicity $[8,11]$. Since vaginal length treated is directly related to volume of vaginal tissue irradiated, one could presume that by reporting TRAK as a treatment intensity parameter, TRAK will play a vital role in studies related to treatment toxicity in VC brachytherapy.

The relationship between TRAK and the total treatment time presented in Eq. 3 is a result of definition of TRAK [12]. This relationship could be utilized as a hand calculation method to quickly verify planned treatment time, knowing the value of TRAK from treatment plan in any HDR brachytherapy application. This method, by itself, does not exactly constitute an independent second-check of the treatment time since the TRAK value input is taken from the treatment plan. However, it does add a value as an additional quick overall check of planned treatment time for a given TRAK value by a physician or a physicist [13]. As presumed in the previous statement, a thorough independent check of the treatment plan by a secondary check method (as required in published QA guidelines) was performed, and our pro-

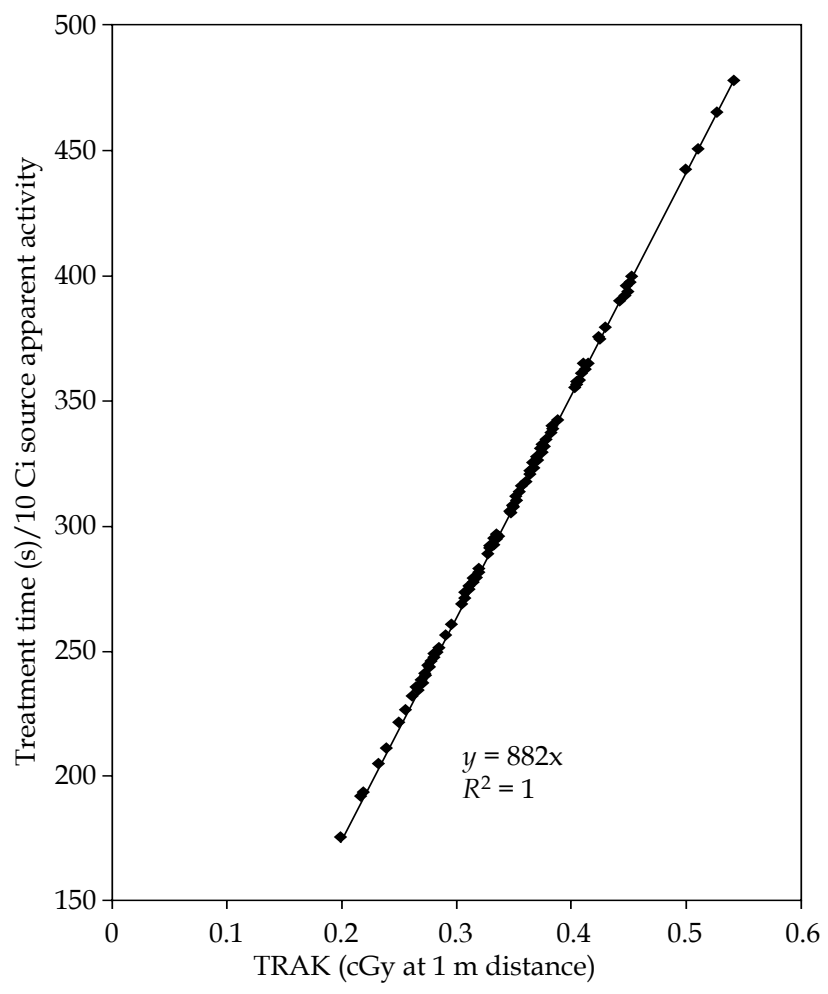

Fig. 3. A scatterplot of TRAK vs. total planned treatment time for 175 vaginal cylinder brachytherapy cases. TRAK - total reference air-kerma (cGy), total treatment time in seconds for a $10 \mathrm{Ci}$ apparent activity of ${ }^{192} \mathrm{Ir}$ source

posed treatment time check only serves as an additional plan QA tool prior to treatment.

Additionally, TRAK parameter could be utilized as a secondary plan quality check parameter to establish a range of optimal TRAK values for specific VC applicator size that could be derived based on individual institutional dose prescription and optimization practices. In our study, the average TRAK values for the cylinder diameters utilized were: 0.23 (size $20 \mathrm{~mm}$ ), 0.27 (size $25 \mathrm{~mm}$ ), 0.32 (size $30 \mathrm{~mm}$ ), 0.38 (size $35 \mathrm{~mm}$ ), and 0.46 (size $40 \mathrm{~mm}$ ). Ideally, one would expect a fixed value of TRAK for a given VC size, dose, length, and fixed depth of prescription. However, differences in TRAK values exist depending on the method used for dose optimization, such as lateral dose points only or lateral dose points plus accounting for curvature of cylinder dome. These differences could result in different volumes irradiated by the prescription dose [10].

\section{Conclusions}

The correlation of TRAK to the total volume encompassed by the prescription dose surface in a single-channel vaginal cylinder brachytherapy yields a useful predictive equation, but restrictive in application due to differences in dose optimization schemes, implemented for vaginal cylinder HDR brachytherapy treatment that vary in clinical practice. However, the linear correlation observed between TRAK and the volume of vaginal tissue irradiated, could be of clinical interest in treatment 
toxicity-related studies. The TRAK treatment time relationship enables quick verification of planned treatment time by knowing TRAK in any HDR brachytherapy application.

\section{Disclosure}

The authors report no conflict of interest.

\section{References}

1. International Commission on Radiation Units and Measurements (ICRU). Prescribing, recording, and reporting brachytherapy for cancer of the cervix. ICRU Report 89, New York 2016

2. Wilkinson JM, Ramachandran TP. The ICRU recommendations for reporting intracavitary therapy in gynecology and the Manchester method of treating cancer of the cervix uteri. Br J Radiol 1989; 62: 362-365.

3. Eisbruch A, Williamson JF, Dickson DR et al. Estimation of tissue volume irradiated by intracavitary implants. Int J Radiat Oncol Biol Phys 1993; 25: 733-744.

4. Deshpande DD, Shrivatsava SK, Nehru RM et al. Treatment volume from total reference air kerma (TRAK) in intracavitary applications and its composition with ICRU reference volume. Int J Radiat Oncol Biol Phys 1994; 28: 499-504.

5. Datta NR, Srivastava A, Maria Das KJ et al. Total reference air kerma: To what extent can it predict intracavitary volume enclosed by isodose surfaces during multiple high-dose rate brachytherapy? Brachytherapy 2003; 2: 91-97.

6. Nkiwane KS, Andersen E, Champoudry J et al. Total reference air kerma can accurately predict isodose surface volumes in cervix cancer brachytherapy. A multicenter study. Brachytherapy 2017; 16: 1184-1191.

7. Robert N, Chauhan RP, Oinam A, Rai B. Formulation of normal tissue irradiation volumes in Co-60 and Ir-192 HDR ICBT of Ca cervix using Total Reference Air Kerma (TRAK). Rep Pract Oncol Radiother 2019; 24: 568-575.

8. Guy CL, Fields EC, Quinn BA et al. The vaginal cylinder: misunderstood, misused or trivial? An in-depth dosimetric and multi-institutional outcome investigation. Brachytherapy 2019; 18: 763-770.

9. Serban M, Kirisits C, Potter R et al. Isodose surface volumes in cervix cancer brachytherapy: Change of practice from standard (Point A) to individualized image guided adaptive (EMBRACE I) brachytherapy. Radiother Oncol 2018; 129: 567-574.

10. Somangili SS, Johnson GRS, Supe SS et al. Optimization in high dose rate vaginal cylinder for vaginal cuff irradiation. Rep Pract Oncol Radiother 2008; 13: 35-48.

11. Delishaj D, Barcellini A, D'Amico R et al. Vaginal toxicity after high-dose-rate endovaginal brachytherapy: 20 years of results. J Contemp Brachytherapy 2018; 10: 559-566.

12. Williamson JF, Coursey BM, DeWerd LA et al. On the use of apparent activity $\left(\mathrm{A}_{\text {app}}\right)$ for treatment planning of ${ }^{125} \mathrm{I}$ and ${ }^{103} \mathrm{Pd}$ interstitial brachytherapy sources: recommendations of the American Association of Physicist in Medicine Radiation Therapy Committee Subcommittee on Low-Energy Brachytherapy Source Dosimetry. Med Phys 1999; 26: 2529-2530.

13. Kubo DH, Glasgow GP, Pethel TD et al. High dose-rate brachytherapy treatment delivery: Report of the AAPM Radiation Therapy Committee Task Group No. 59. Med Phys 1998; 25: 375-403. 